\title{
Monte Carlo evaluation of the continuum limit of the two-point function of two Euclidean Higgs real scalar fields subject to affine quantization
}

\author{
Riccardo Fantoni $\circledast^{1, *}$ and John R. Klauder $\circledast^{2, \dagger}$ \\ ${ }^{1}$ Università di Trieste, Dipartimento di Fisica, strada Costiera 11, 34151 Grignano (Trieste), Italy \\ ${ }^{2}$ Department of Physics and Department of Mathematics University of Florida, \\ Gainesville, Florida 32611-8440, USA
}

(Received 23 July 2021; accepted 13 September 2021; published 29 September 2021)

\begin{abstract}
We study canonical and affine versions of the quantized covariant Euclidean Higgs scalar field-theory for two real fields on four dimensional lattices through the Monte Carlo method. We calculate the two-point function near the continuum limit at finite volume.
\end{abstract}

DOI: 10.1103/PhysRevD.104.054514

\section{INTRODUCTION}

The aim of this work is to find out what affine quantization $[1,2]$ does to a classical field-theory for two real scalar fields, or equivalently a complex scalar field, of mass $m$ subject to the Mexican-hat, Higgs potential, rather than canonical quantization [3]. To this aim we will compare the two-point function of the two fields in the two frameworks.

In particular in this paper we try to understand in what ways an affine quantization is similar as well as dissimilar from a canonical quantization. We add that some nonfree real scalar fields have already been observed and that canonical quantization fails for several nonrenormalizable fields, such as $\left(\phi^{12}\right)_{3}$ [4] and $\left(\phi^{4}\right)_{4}$ [5]. The key to that result is the introduction of a highly unusual, additional, nonquadratic, term that is dictated by affine quantization. While affine quantization employs an additional term, that particular term formally disappears when the Planck constant $\hbar \rightarrow 0$, which makes it a plausible modification of the quadratic terms of traditional free real scalar fields in order to extend acceptable quantization of traditional nonrenormalizable models. [6-10]

This work should be considered as a follow up of our previous work [11] where the two-point function of a single Euclidean free real scalar field subject to affine quantization was found through Monte Carlo (MC) methods. In particular in that work we found that the vacuum expectation value of the field diverges in the continuum limit. This

\footnotetext{
riccardo.fantoni@posta.istruzione.it

klauder@ufl.edu
}

Published by the American Physical Society under the terms of the Creative Commons Attribution 4.0 International license. Further distribution of this work must maintain attribution to the author(s) and the published article's title, journal citation, and DOI. Funded by SCOAP ${ }^{3}$. shortcoming is expected to disappear in the present case of a complex field $\varphi=\phi_{1}+i \phi_{2}$. In fact, in this case, one can go "slowly" "around" the peak at $\varphi=0$ with no need of "jumps" [11].

The covariant Euclidean action in canonical quantization [3] is ${ }^{1}$

$$
\begin{aligned}
S^{(c)}\left[\phi_{1}, \phi_{2}\right]= & \int\left\{\frac{1}{2} \sum_{\mu=0}^{s}\left[\left(\frac{\partial \phi_{1}(x)}{\partial x_{\mu}}\right)^{2}+\left(\frac{\partial \phi_{2}(x)}{\partial x_{\mu}}\right)^{2}\right]\right. \\
& \left.+V\left(\phi_{1}(x), \phi_{2}(x)\right)\right\} d^{n} x, \\
V\left(\phi_{1}, \phi_{2}\right)= & \frac{1}{2} m^{2}\left(\phi_{1}^{2}+\phi_{2}^{2}\right)+g\left[\left(\phi_{1}^{2}+\phi_{2}^{2}\right)-\Phi^{2}\right]^{2},
\end{aligned}
$$

with $x=\left(x_{0}, x_{1}, \ldots, x_{s}\right)=\left(x_{0}, \vec{x}\right)$ for $s$ spatial dimensions and $n=s+1$ for the number of space-time dimensions with $x_{0}=c t$, where $c$ is the speed of light constant and $t$ extends from zero to $\hbar \beta$ with $\beta=1 / k_{B} T, k_{B}$ being the Boltzmann constant and $T$ the absolute temperature. We will work at $s=3$. And $V$ is the self-interaction potential density corresponding to an interacting Higgs theory with a bare mass $m$ and a bare coupling $g$.

The covariant Euclidean action in affine quantization $[1,2]$ is

$$
\begin{aligned}
S^{(a)}\left[\phi_{1}, \phi_{2}\right] & =\int\left\{\frac{1}{2} \sum_{\mu=0}^{s}\left[\left(\frac{\partial \phi_{1}(x)}{\partial x_{\mu}}\right)^{2}+\left(\frac{\partial \phi_{2}(x)}{\partial x_{\mu}}\right)^{2}\right]\right. \\
& \left.+\frac{3}{8} \frac{\delta^{2 s}(0) \hbar^{2}}{\phi_{1}^{2}(x)+\phi_{2}^{2}(x)+\epsilon}+V\left(\phi_{1}(x), \phi_{2}(x)\right)\right\} d^{n} x,
\end{aligned}
$$

\footnotetext{
${ }^{1}$ Note however that Eq. (1.3) can be simplified to $V=g\left[\left(\phi_{1}^{2}+\phi_{2}^{2}\right)-A^{2}\right]^{2}+$ constant, where $A$ involves a combination of $\Phi$ and $m: A^{2}=\Phi^{2}-m^{2} / 4 g$.
} 
where $\epsilon>0$ is a parameter used to regularize the " $3 / 8$ " extra term stemming from considering the complex field $\varphi(x)=$ $\phi_{1}(x)+i \phi_{2}(x)$ and the momentum field $\pi(x)=$ $-i \hbar \partial / \partial \varphi(x)$ as the two conjugate canonical variables (see Appendix A in [4]) and $\delta$ is a Dirac delta function. In this case the Hamiltonian density formally contains a divergent term, ${ }^{2}$ in the total potential density $\mathcal{V}(\phi)=\frac{3}{8} \delta^{2 s}(0) \hbar^{2} /$ $\left(\phi_{1}^{2}+\phi_{2}^{2}+\epsilon\right)+V(\phi)$, in the continuum, but the field theory can be regularized and treated on a lattice, and the approach toward the continuum will be taken under exam in this work. In the following we will use natural units with $c=\hbar=k_{B}=1$.

In our previous works we studied the single real scalar field nonrenormalizable canonical cases with $V(\phi)=\frac{1}{2} m^{2} \phi^{2}+$ $g \phi^{4}[5]$ in $s=3$ and $\frac{1}{2} m^{2} \phi^{2}+g \phi^{12}$ in $s=2$ [4], where $g$ is the bare coupling constant. And we showed that the corresponding affine cases are indeed renormalizable.

MC [12,13] is the numerical method of choice to treat multidimensional integrals of high dimensions and, therefore, is especially useful to compute path integrals. We will use it to study the two-point function of the Euclidean action of two real scalar field in affine quantization. Our estimate of the path integrals will be generally subject to three sources of numerical uncertainties: The one due to the statistical errors, the one due to the space-time discretization, and the one due to the finite-size effects. Of these, the statistical errors scale like $M^{-1 / 2}$ where $M$ is the computer time, the discretization of space-time is responsible for the distance from the continuum limit (which corresponds to a lattice spacing $a \rightarrow 0$ ), and the finite-size effects stems from the necessity to approximate the infinite space system with one in a periodic box of volume $L^{s}$ with $L=N a$ being the box side, subject to $N$ discretization points. The finitesize effects are due to the distance from the thermodynamic limit (which corresponds to $N \rightarrow \infty$ ). [14]

The work is organized as follows: In Sec. II we derive the lattice formulation of the field theory needed in the treatment on the computer; in Sec. III we describe our computer experiment and introduce the observables that will be measured during our simulations; in Sec. IV we present our partial results obtained by working with the two scalar fields $\phi_{1}$ and $\phi_{2}$ where we encounter ergodicity problems for the affine case; in Sec. V we are able to overcome the ergodicity breakdown observed in the previous section and we present our final results for the affine case obtained by working with the two scalar fields $\rho=\sqrt{\phi_{1}^{2}+\phi_{2}^{2}}$ and $\theta=$ $\arctan \left(\phi_{2} / \phi_{1}\right)$ such that $d \phi_{1} d \phi_{2}=\rho d \theta d \rho$. Section VI is for final remarks.

\footnotetext{
${ }^{2}$ The divergent integral $\int_{-N}^{N} d \phi / \phi^{2}$, can be made finite simply by a regularized integral such as $\int \sum_{n=-N}^{\prime N}\left(1 / 3 r^{2}\right)\left[(n+1)^{2} \cos ^{2}(k)+\right.$ $\left.n^{2} \sin ^{2}(k) \cos ^{2}\left(k^{\prime}\right)+(n-1)^{2} \sin ^{2}(k) \sin ^{2}\left(k^{\prime}\right)\right]^{-1} d r^{3} d k d k^{\prime}$ where the prime over the sum indicates that we are considering a periodic closure $-N, N$ for the three terms in square brackets.
}

\section{THE LATTICE FORMULATION OF THE FIELD-THEORY MODEL}

We used a lattice formulation of the field theory. The theory considers a complex scalar field $\varphi=\phi_{1}+i \phi_{2}$ taking the value $\varphi(x)$ on each site of a periodic, hypercubic, $n$-dimensional lattice of lattice spacing $a$ and periodicity $L=N a$. The canonical covariant action for the field, Eq. (1.1), is then approximated by

$$
\begin{aligned}
\frac{S^{(c)}\left[\phi_{1}, \phi_{2}\right]}{a^{n}} \approx & \frac{1}{2 a^{2}} \sum_{x, \mu}\left\{\left[\phi_{1}(x)-\phi_{1}\left(x+e_{\mu}\right)\right]^{2}\right. \\
& \left.+\left[\phi_{2}(x)-\phi_{2}\left(x+e_{\mu}\right)\right]^{2}\right\} \\
& +\sum_{x} V\left(\phi_{1}(x), \phi_{2}(x)\right),
\end{aligned}
$$

where $e_{\mu}$ is a vector of length $a$ in the $+\mu$ direction and we are at a temperature $T=1 / N a$, in units where Boltzmann constant $k_{B}=1$.

Note that in our model the continuous symmetry $\varphi \rightarrow e^{i \alpha} \varphi$ breaks down spontaneously and the mass spectrum contains a Goldstone boson. The accepted signal of a system being in the symmetry broken phase in a finite volume, in the absence of a small symmetry breaking term, is not a nonzero order parameter, but rather the fact that a product of order parameters, at points $x, y$, tends to a nonzero limit with increasing $|x-y|$. To understand the properties of the system at finite volume, it is convenient to add a small symmetry breaking term and to work with the potential

$$
V=g\left(\phi_{1}^{2}+\phi_{2}^{2}-A^{2}\right)^{2}+\left(\varepsilon^{2} / 2\right) \phi_{2}^{2}+\text { constant },
$$

The term proportional to $\varepsilon^{2}$ ensures that the classical action has a proper minimum at the point $\phi_{1}=A, \phi_{2}=0$. The expansion of the potential in powers of $\psi=\phi_{1}-A$, and $\phi_{2}$ starts with

$$
\begin{gathered}
V=\left(M^{2} / 2\right) \psi^{2}+\left(\varepsilon^{2} / 2\right) \phi_{2}^{2}+\ldots, \\
M=A \sqrt{8 g}
\end{gathered}
$$

The first term represents a free particle of mass $M$, the second a free particle of mass $\varepsilon$. The situation is the same as in the case of the free real scalar field: the perturbative expansion of the two-point function starts with

$$
\begin{gathered}
\left\langle\phi_{1}(x) \phi_{1}(y)\right\rangle=A^{2}+D(x-y, M, L), \\
\left\langle\phi_{2}(x) \phi_{2}(y)\right\rangle=D(x-y, \varepsilon, L), \\
\left\langle\phi_{1}(x) \phi_{2}(y)\right\rangle=0,
\end{gathered}
$$

where $\langle\ldots\rangle$ is the vacuum expectation value [defined in Eq. (2.11)] and $D(z, m, L)$ is the propagator of a free particle 
of mass $m$ on a hypercubic Euclidean box of size $L^{n}$. For $\varepsilon=0$, the term $D(z, \varepsilon, L)$ reduces to a sum of free massless propagators:

$$
\begin{aligned}
& D(z, 0, L) \\
& =\left(1 / 4 \pi^{2}\right) \sum_{n_{0}, n_{1}, n_{2}, n_{3}} 1 /\left[\left(z_{0}+n_{0} L\right)^{2}+\cdots+\left(z_{3}+n_{3} L\right)^{2}\right],
\end{aligned}
$$

where $z=\left(z_{0}, z_{1}, \ldots, z_{s}\right)$ and $n_{\mu} \in \mathbb{Z}$ for $\mu=0,1, \ldots, s$, but this expression does not make sense because the sum diverges. As long as $\varepsilon$ is different from zero, the limit $L \rightarrow \infty$ ensures that a single term in the sum survives, the one with $n_{0}=\ldots=n_{3}=0$, which describes the contribution from the Goldstone boson.

Expression (2.1) needs to be modified for the affine action of Eq. (1.3). In this case the Dirac delta function is replaced by $\delta^{2 s}(0) \rightarrow a^{-2 s}$. Moreover it is convenient the following scaling: $\phi_{i}=a^{-s / 2} \bar{\phi}_{i}, \Phi_{i}=a^{-s / 2} \bar{\Phi}_{i}, g=a^{s} \bar{g}$, and $\epsilon=a^{-s} \bar{\epsilon}$ which gives the following discretized approximation for the affine action

$$
\begin{aligned}
\frac{S^{(a)}\left[\bar{\phi}_{1}, \bar{\phi}_{2}\right]}{a^{-s} a^{n} \approx} & \frac{1}{2 a^{2}} \sum_{x, \mu}\left\{\left[\bar{\phi}_{1}(x)-\bar{\phi}_{1}\left(x+e_{\mu}\right)\right]^{2}+\left[\bar{\phi}_{2}(x)-\bar{\phi}_{2}\left(x+e_{\mu}\right)\right]^{2}\right\} \\
& +\sum_{x} \frac{3}{8} \frac{1}{\bar{\phi}_{1}^{2}(x)+\bar{\phi}_{2}^{2}(x)+\bar{\epsilon}} \\
& +\sum_{x}\left\{\frac{1}{2} m^{2}\left(\bar{\phi}_{1}^{2}(x)+\bar{\phi}_{2}^{2}(x)\right)+\bar{g}\left[\left(\bar{\phi}_{1}^{2}(x)+\bar{\phi}_{2}^{2}(x)\right)-\bar{\Phi}^{2}\right]^{2}\right\} .
\end{aligned}
$$

Note that if $g$ is taken different from zero, the relation $g=a^{s} \bar{g}$ shows that $\bar{g}$ carries a dimension. Setting $\bar{g}=M^{s}$, $M$ is of dimension mass (we are using natural units $c=\hbar=1$ ). If $M$ as well as $m$ are kept fixed when the cutoff is removed, the model contains the two dimensionful parameters $m$ and $M$. The lattice spacing $a$ must be small compared to $1 / \mathrm{m}$ as well as compared to $1 / M$ and the box must be large compared to $1 / M$. Since $\bar{\phi}$ is of dimension mass $^{-1 / 2}$, the two-point function of $\bar{\phi}$ is of the form

$$
\left\langle\bar{\phi}_{i}(x) \bar{\phi}_{j}(y)\right\rangle=f_{i j}\{M(x-y), m / M, a M, L M\} / M .
$$

To approach the continuum limit, the last two argument must be in the range: $a M \ll 1, L M \ll 1$. The only relevant parameter, apart from the number of lattice points, used to regularize the system should be the ratio $m / M$.

We will use the so called "primitive approximation" for the action [see Eqs. (2.1) or (2.9)] even if it can be improved in several ways [15] in order to reduce the error due to the space-time discretization. In reaching to the expression (2.1) or (2.9) we neglected the term $\propto a^{2 n}$ due to the commutator of the kinetic and potential parts of the Hamiltonian, in the BakerCampbellHausdorff formula. In reaching to the path integral expression this is justified by the Trotter formula.

The vacuum expectation of a functional observable $\mathcal{O}\left[\phi_{1}, \phi_{2}\right]$ is

$$
\langle\mathcal{O}\rangle \approx \frac{\int \mathcal{O}\left[\phi_{1}, \phi_{2}\right] \exp \left(-S\left[\phi_{1}, \phi_{2}\right]\right) \prod_{x} d \phi_{1}(x) d \phi_{2}(x)}{\int \exp \left(-S\left[\phi_{1}, \phi_{2}\right]\right) \prod_{x} d \phi_{1}(x) d \phi_{2}(x)},
$$

for a given action $S$.

We will approach the continuum limit by choosing a fixed $L$ and increasing the number of discretizations $N$ of each component of the space-time. So that the lattice spacing $a=L / N \rightarrow 0$. To make contact with the continuum limit, two conditions must be met $a \ll 1 / m \ll L$ where $1 / m$ is the Compton wavelength.

\section{SIMULATION DETAILS AND RELEVANT OBSERVABLES}

We want to determine the two-point function

$$
K_{i j}(x, y)=\left\langle\phi_{i}(x) \phi_{j}(y)\right\rangle,
$$

where in the affine case we need to replace the fields $\phi_{i}$ by the scaled fields $\bar{\phi}_{i}$. Replacing $x$ by $x+k$ with $k=a w_{n}$ with $w_{n}=\left(n_{0}, n_{1}, \ldots, n_{s}\right)$ and $n_{\mu} \in \mathbb{Z}$ amounts to a mere relabeling of the lattice points. Hence, due to translational invariance, $K(x, y)$ can only depend on the difference between the coordinates of the two points and we can define,

$$
D_{i j}(z)=\frac{1}{L^{n}} \sum_{x} K_{i j}(x, x+z) a^{n} .
$$

Moreover due to the symmetry $1 \leftrightarrow 2$ we will have $D_{11}=$ $D_{22} \equiv D_{\text {like }}$ and $D_{12}=D_{21} \equiv D_{\text {unlike. }}$. In our simulations we 
work in periodic space-time (at a temperature $T=1 / N a$ ) so that $\phi_{i}\left(x_{\mu}+N\right)=\phi_{i}\left(x_{\mu}\right)$ for any $x, \mu=0,1, \ldots, s$, and $i=1,2$.

Our MC simulations use the Metropolis algorithm $[12,13]$ to calculate the ensemble average of Eq. (2.11) which is a $2 N^{n}$ multidimensional integral. The simulation is started from the initial condition $\phi_{i}=0$ for $i=1,2$. One MC step consisted in a random displacement of each one of the $2 N^{n}$ variables $\phi_{i}(x)$ for $i=1,2$, as follows

$$
\phi_{i} \rightarrow \phi_{i}+(2 \eta-1) \delta
$$

where $\eta$ is a uniform pseudo random number in $[0,1]$ and $\delta$ is the amplitude of the displacement. The fields $\phi_{i} \in(-\infty, \infty)$ for $i=1,2$ and $x_{\mu} \in[0, L]$ for $\mu=0,1, \ldots, s$. Each one of these $2 N^{n}$ moves is accepted if $\exp (-\Delta S)>\eta$ where $\Delta S$ is the change in the action due to the move (it can be efficiently calculated considering how the kinetic part and the potential part change by the displacement of a single $\phi_{i}(x)$ ) and rejected otherwise. The amplitude $\delta$ is chosen in such a way to have acceptance ratios as close as possible to $1 / 2$ and is kept constant during the evolution of the simulation. One simulation consisted of $M \mathrm{MC}$ steps each of which consisted in a sweep of $2 N^{n}$ displacement moves of all the fields variables. The statistical error on the average $\langle\mathcal{O}\rangle$ will then depend on the correlation time necessary to decorrelate the property $\mathcal{O}, \tau_{\mathcal{O}}$, and will be determined as $\sqrt{\tau_{\mathcal{O}} \sigma_{\mathcal{O}}^{2} /\left(M 2 N^{n}\right)}$, where $\sigma_{\mathcal{O}}^{2}$ is the intrinsic variance for $\mathcal{O}$.

\section{SIMULATION RESULTS}

We worked in units where $c=\hbar=k_{B}=1$. We chose the regularization parameter of the affine quantization term to be $\epsilon=10^{-10}$. $^{3}$

In Fig. 1 we show $D_{\text {like }}(z)$ and $D_{\text {unlike }}(z)$ as obtained for $m=1, g=1, \Phi=1, L=3$ and three choices of $N$, in the canonical scenario. One can then see the approach to the continuum of the two-point functions of the canonical model. From the figure we can see that the unlike two-point function is zero over the whole space-time volume. This can be explained observing that during the random-walk the field will be localized around the minima of the potential density so that $\phi_{1}^{2}+\phi_{2}^{2} \approx \Pi_{c}^{2}$ with $\Pi_{c}$ the radius of the minima ring, the circle of vacua, around the origin $\varphi=0$, which is a function of $m, g$, and $\Phi$ :

$$
\Pi_{c}^{2}=\frac{4 g \Phi^{2}-m^{2}}{4 g} .
$$

So that the Higgs potential density in the action does not actually contribute to correlate the two fields $\phi_{i}$ for $i=1,2$.

\footnotetext{
${ }^{3}$ Note that we could as well choose a regularization putting hard walls at $\phi_{i}= \pm \varepsilon$ therefore rejecting MC moves whenever $\phi_{i} \in[-\varepsilon, \varepsilon]$, for $i=1,2$.
}
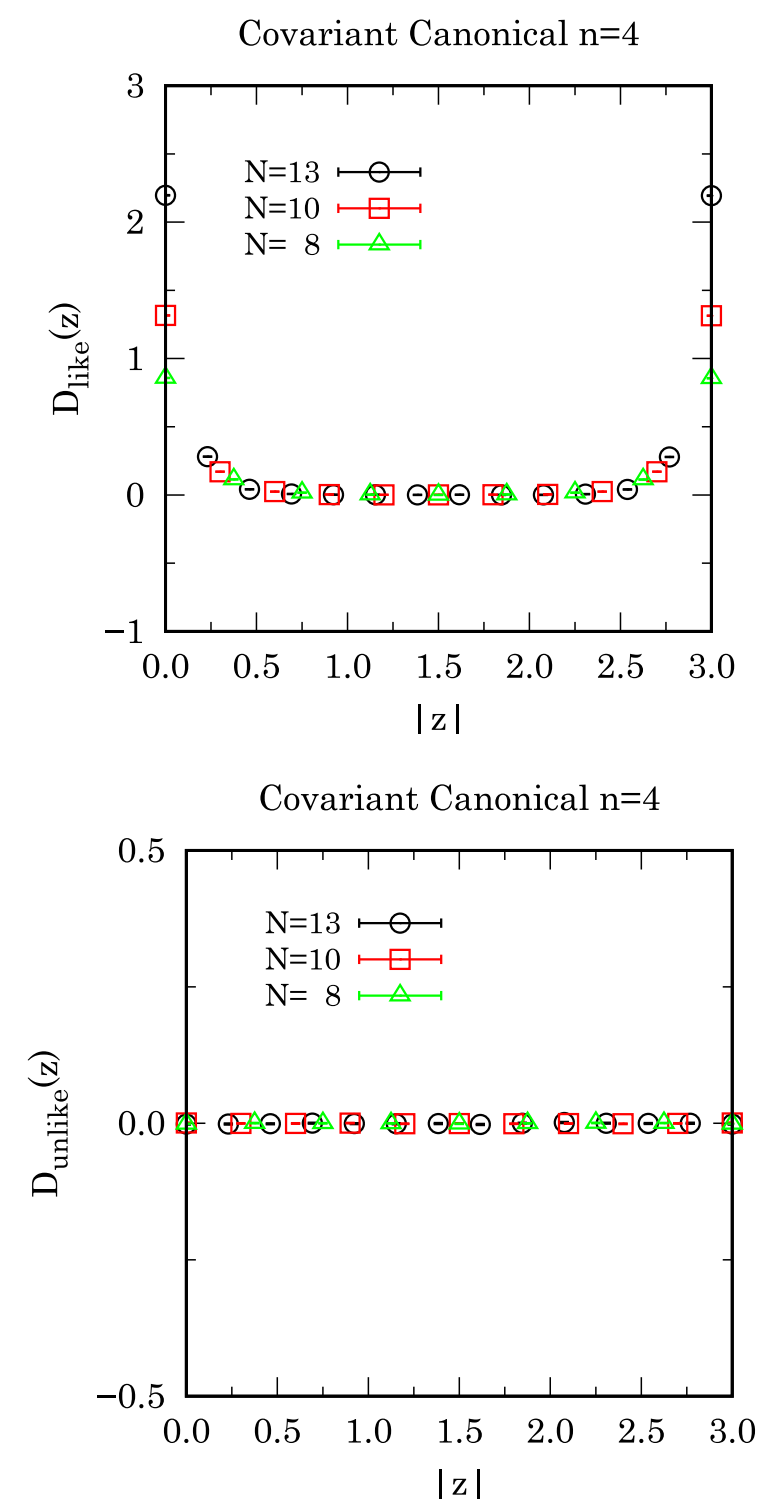

FIG. 1. Two-point functions, $D_{\text {like }}(z)$ (top panel) and $D_{\text {unlike }}(z)$ (bottom panel), of Eq. (3.2) for the complex scalar Higgs field $\varphi=\phi_{1}+i \phi_{2}$ subject to canonical quantization with a selfinteraction potential density of the form $V=\frac{1}{2} m^{2}\left(\phi_{1}^{2}+\phi_{2}^{2}\right)+$ $g\left(\phi_{1}^{2}+\phi_{2}^{2}-\Phi^{2}\right)^{2}$ in Eq. (1.3) with $m=1, g=1, \Phi=1, L=3$ $\left(\Pi_{c}^{2}=3 / 4\right)$ and increasing $N=8,10,13$. On the abscissa axis we have $|z|=\sqrt{z_{0}^{2}+z_{1}^{2}+\cdots+z_{s}^{2}}$ which is a length.

Moreover, the expectation values $\left\langle\phi_{i}\right\rangle=0$ for $i=1,2$ because the complex field $\varphi$ tends to rotate around the origin on the minima ring. The approach to the continuum is manifested through increasing values of $D_{\text {like }}(0)$ with increasing $N$. For our choice of the parameters $m^{2}<4 g \Phi^{2}$ and we must have symmetry breaking [8-10], with the circle of vacua having a radius different from zero. The renormalized coupling constant [5] was found to be: $g_{R}=$ $-0.0069(6)$ for $N=8, g_{R}=-0.0006(4)$ for $N=10, g_{R}=$ +0.0000 (5) for $N=13$. Since $g_{R}$ must be non-negative, by 
Lebowitz inequality, our results signal a free trivial system in the continuum limit.

For the affine quantization case the circle of vacua has a radius $\bar{\Pi}_{a}$, which is now a function of $m, \bar{g}$, and $\bar{\Phi}$ :

$$
\begin{aligned}
\bar{\Pi}_{a}^{2}= & \frac{4 \bar{g} \bar{\Phi}^{2}-m^{2}}{12 \bar{g}} \\
& +\frac{\left(4 \bar{g} \bar{\Phi}^{2}-m^{2}\right)^{2}}{12 \bar{g}\left[162 \bar{g}^{2}+\Xi+18 \bar{g}^{2 / 3}\left(81 \bar{g}^{2}+\Xi\right)^{1 / 3}\right]} \\
& +\frac{162 \bar{g}^{2}+\Xi+18 \bar{g}^{2 / 3}\left[81 \bar{g}^{2}+\Xi\right]^{1 / 3}}{12 \bar{g}}, \\
\Xi & =-m^{6}+12 \bar{g} m^{4} \bar{\Phi}^{2}-48 \bar{g}^{2} m^{2} \bar{\Phi}^{4}+64 \bar{g}^{3} \bar{\Phi}^{6},
\end{aligned}
$$

where without loss of generality we assumed $\epsilon=0$. It is different from zero irrespectively from the values of the parameters, so symmetry is always broken. In Fig. 2 we show $D_{\text {like }}(z)$ and $D_{\text {unlike }}(z)$ as obtained for $m=1, \bar{g}=$ $1, \bar{\Phi}=1, L=3$ (so that $m / M=1$ ), $\epsilon=10^{-10}$ (the simulation results are not affected by $\epsilon$ as long as it is chosen sufficiently small), and three choices of $N$, in the affine scenario, for the $\bar{\phi}_{i}$ fields introduced in Eq. (2.9). One can then see the approach to the continuum of the two-point functions of the affine model. Note, however, that now the region around $\bar{\phi}_{i}=0$ for $i=1,2$ is forbidden due to the affine $3 / 8$ diverging term in the potential density [see Eq. (2.9)], therefore the complex field in its "winding" around the origin, in proximity of the potential minima ring, cannot take a "shortcut" through the "mountain" at the origin (the forbidden region) and this, in turn, is responsible for a loss of ergodicity and the appearance of systematic errors in addition to the usual statistical ones. It is then necessary an extremely long simulation (much longer than the average time for a "round trip" of the field), much longer than in the canonical case. Notice, moreover, that the action is penalized by the additional $a^{-s}$ factor which grows as we approach the continuum $a \rightarrow 0$. A possible solution would be to choose the field displacement $\delta$ larger than the diameter of the potential minima ring $2 \bar{\Pi}_{a}$. But unfortunately this will not work because the kinetic energy term in the action doesn't allow the field to undergo big "jumps." In addition this would generate low acceptance ratios thereby slowing down the simulation. An alternative solution will be given in the next section. In our simulations, that were $M=10^{7} \mathrm{MC}$ steps long, the expectation value of the field $\left\langle\bar{\phi}_{1}\right\rangle=\left\langle\bar{\phi}_{2}\right\rangle$ was equal to $-0.23(4)$ for $N=8$, to $-0.24(4)$ for $N=10$, and to $-0.784(9)$ for $N=13$. A nonzero value for the vacuum expectation of the field is due to the systematic errors described above and will eventually disappear in an extremely long simulation. From the figure we see how the two-point like function seems to be increasing with $N$, while the unlike one has a constant behavior fluctuating around the expected zero value. These results are still affected by the ergodicity systematic errors stemming from the "winding" random walk. In order to show this behavior, we calculated the histograms of the values for $\left\langle\bar{\phi}_{1}\right\rangle$ obtained by averaging over blocks of 100 MC steps during the simulation, that we call $H \bar{\phi}$, of $D_{\text {like }}(0)$ that we call $H D_{\text {like }}$, and of $D_{\text {unlike }}(0)$, that we call $H D_{\text {unlike }}$. The behavior of these histograms is shown in Figs. 3-5 respectively. From the histogram of Fig. 3 we see how for $N=13$ the field did not have the chance of rotating around the origin and this explains the lack of the first peak in the histogram of Fig. 4. We then conclude that
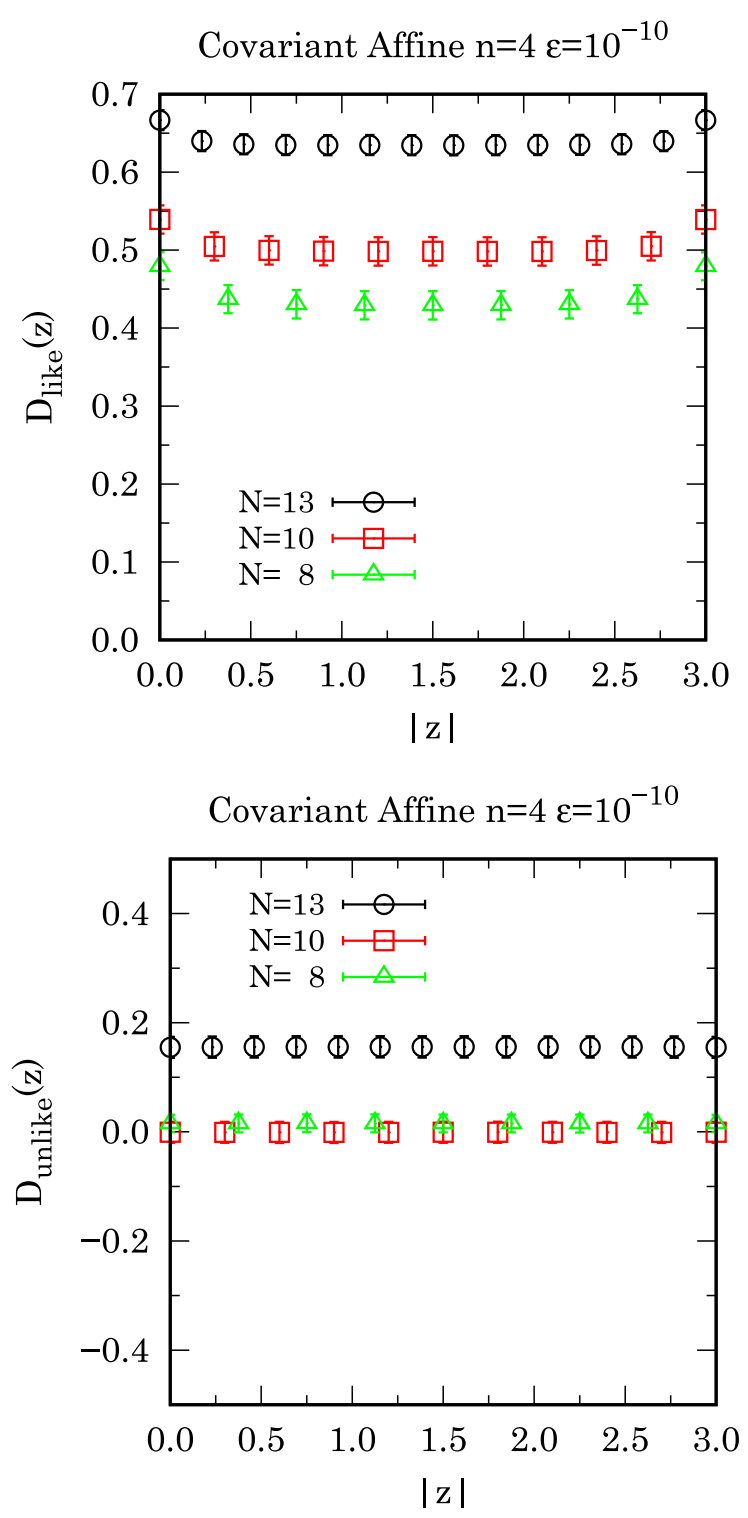

FIG. 2. Two-point functions, $D_{\text {like }}(z)$ (top panel) and $D_{\text {unlike }}(z)$ (bottom panel), of Eq. (3.2) for the complex scalar Higgs field $\bar{\varphi}=\bar{\phi}_{1}+i \bar{\phi}_{2}$ subject to affine quantization with a self-interaction potential density of the form $V=\frac{1}{2} m^{2}\left(\phi_{1}^{2}+\phi_{2}^{2}\right)+g\left(\phi_{1}^{2}+\right.$ $\left.\phi_{2}^{2}-\Phi^{2}\right)^{2}$ in Eq. (1.3) with $m=1, \bar{g}=1, \bar{\Phi}=1, L=3, \epsilon=$ $10^{-10}\left(\bar{\Pi}_{a}^{2} \approx 0.955410\right)$ in Eq. (2.9) and increasing $N=8,10,13$. The simulation used $M=10^{7} \mathrm{MC}$ steps. On the abscissa axis we have $|z|=\sqrt{z_{0}^{2}+z_{1}^{2}+\cdots+z_{s}^{2}}$ which is a length. 


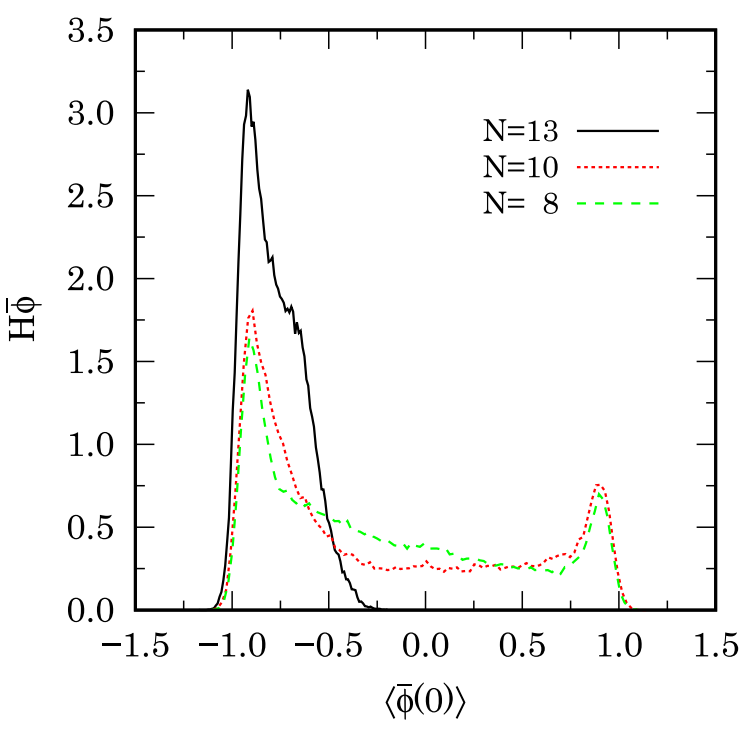

FIG. 3. Histogram of $\left\langle\bar{\phi}_{1}\right\rangle$ block values during the simulation shown in Fig. 2. The figure shows the "rotation" of the field around the origin in proximity of the potential minima ring of radius $\bar{\Pi}_{a} \approx 0.977451$, for $N=8$ and 10 , but not for $N=13$. Even for $N=8$ and 10 the rotation was not symmetric (this would only be obtained in an extremely long simulation), which explains the not exactly zero value of the expectation value of field.

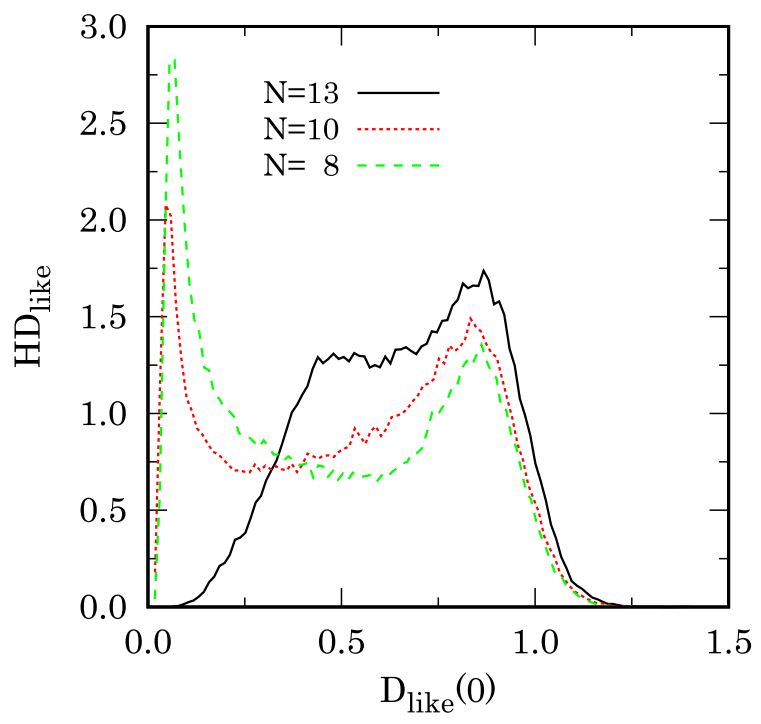

FIG. 4. Histogram of $D_{\text {like }}(0)$ block values during the simulation shown in Fig. 2. The missing first peak in the $N=13$ data is due to the fact that the field did not perform a full rotation around the origin as is shown by Fig. 3 .

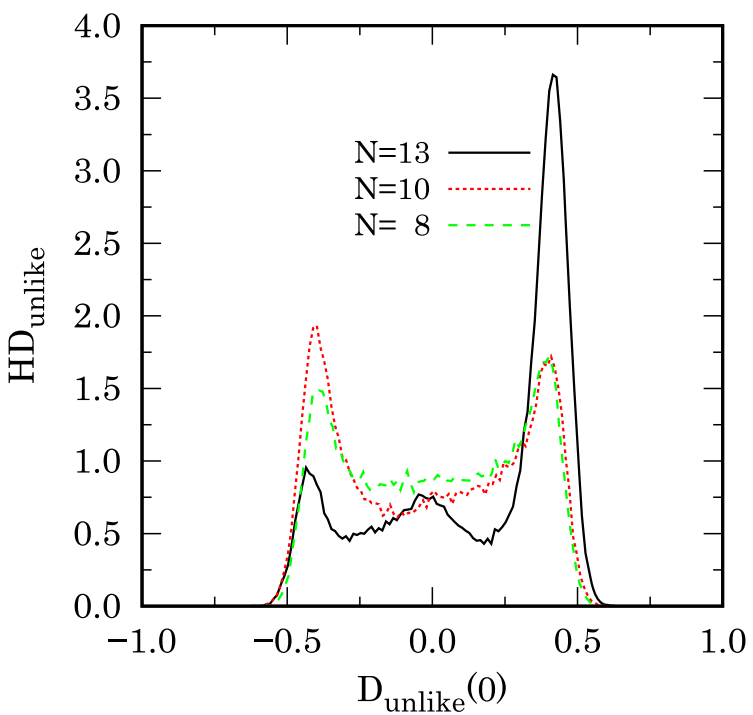

FIG. 5. Histogram of $D_{\text {unlike }}(0)$ block values during the simulation shown in Fig. 2. The $N=13$ data presents a high asymmetry during the evolution of the simulation, which again signals that the simulation was not long enough.

the simulation for $N=13$ was not long enough. And this is responsible for the high value of the two-point like function observed for $N=13$, as shown in Fig. 2. In order to obtain a fully symmetric rotation of the field random walk around the origin we would clearly need an extremely long simulation. Nonetheless from the partial results of our long simulation we can gather a flavor of the convergence of the two-point functions in the affine case in the continuum limit at finite volume.

These results, albeit partial in their nature, give to affine quantization a role as a method producing meaningful quantum field theories even when, as we have already seen in our previous works $[4,5,11]$, the more common canonical quantization fails. Moreover with the scaling used in Eq. (2.9) the field theory does not suffer from the unpleasant feature of a diverging vacuum expectation value of the field in the continuum limit, which was observed in Ref. [11].

\section{EXPONENTIAL REPRESENTATION OF THE COMPLEX FIELD}

In order to solve the ergodicity breakdown problem encountered in the previous section for the affine case we decided to rewrite our path integral in terms of the fields $\rho(x)$ and $\theta(x)$ such that $\bar{\varphi}(x)=\rho(x) \exp [i \theta(x)]$. Equation (2.9) may be rewritten as follows

$$
\begin{aligned}
\frac{S^{(a)}[\rho, \theta]}{a^{-s} a^{n}} \approx & \frac{1}{2 a^{2}} \sum_{x, \mu}\left\{\left[\rho(x)-\rho\left(x+e_{\mu}\right)\right]^{2}+\rho^{2}(x)\left[\theta(x)-\theta\left(x+e_{\mu}\right)\right]^{2}\right\} \\
& +\sum_{x}\left\{\frac{3}{8} \frac{1}{\rho^{2}(x)+\bar{\epsilon}}+\frac{1}{2} m^{2} \rho^{2}(x)+\bar{g}\left[\rho^{2}(x)-\bar{\Phi}^{2}\right]^{2}\right\},
\end{aligned}
$$



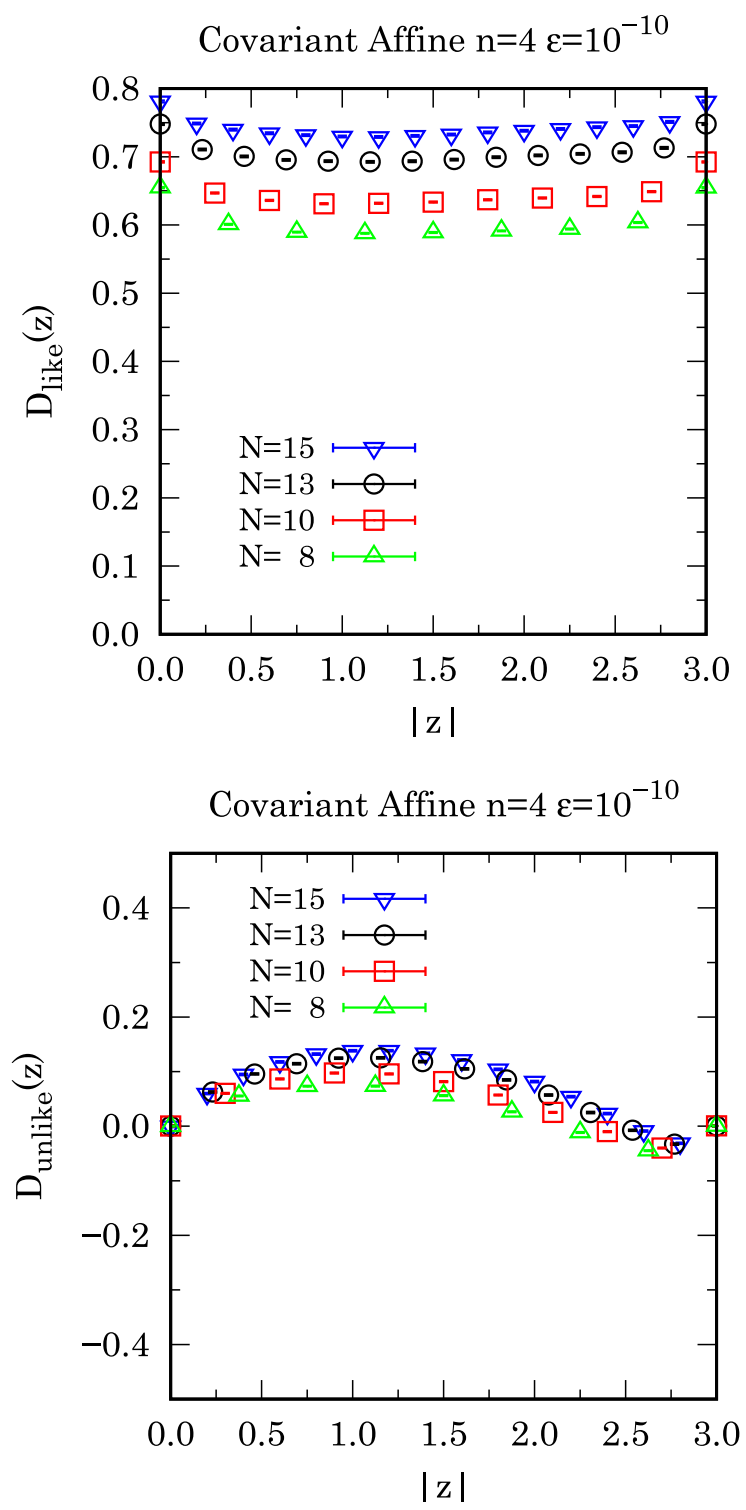

FIG. 6. Two-point functions, $D_{\text {like }}(z)$ (top panel) and $D_{\text {unlike }}(z)$ (bottom panel), of Eq. (3.2) for the complex scalar Higgs field $\bar{\varphi}=\bar{\phi}_{1}+i \bar{\phi}_{2}=\rho \exp (i \theta)$ subject to affine quantization with a self-interaction potential density of the form $V=\frac{1}{2} m^{2} \rho^{2}+$ $g\left(\rho^{2}-\Phi^{2}\right)^{2}$ in Eq. (1.3) with $m=1, \bar{g}=1, \bar{\Phi}=1, L=3, \epsilon=$ $10^{-10}$ in Eq. (5.1) and increasing $N=8,10,13,15$. The simulation used $M=10^{6} \mathrm{MC}$ steps. On the abscissa axis we have $|z|=\sqrt{z_{0}^{2}+z_{1}^{2}+\cdots+z_{s}^{2}}$ which is a length.

and the path integral over $\rho \in[0, \infty]$ and $\theta \in[-\infty, \infty]$ will not suffer anymore from the ergodicity problem. In the Metropolis algorithm we will now have acceptance when $\exp \left[-\left(S^{\prime}-S\right)\right] \prod_{x} \rho^{\prime}(x) / \rho(x)>\eta$ where the primed quantities are the newly generated ones and as usual $\eta$ is a pseudo random number in $[0,1]$. The modulus displacement move, $\rho \rightarrow \rho^{\prime}=\rho+(2 \eta-1) \delta_{\rho}$, is rejected whenever $\rho^{\prime}<0$. And the argument displacement move is chosen purposely asymmetric, $\theta \rightarrow \theta^{\prime}=\theta+\eta \delta_{\theta}$, in order to allow for the required rotation and break the symmetry. This transition rule for the argument will not violate the detailed balance, required by the Metropolis algorithm, as long as the maximum displacement is chosen $\delta_{\theta} \geq 2 \pi$ so that the probability to go from an angle $\theta_{A}$ to $\theta_{B}$ will be equal to the one to return to $\theta_{A}$ from $\theta_{B}$ always using counterclockwise rotations.

In Fig. 6 we show $D_{\text {like }}(z)$ and $D_{\text {unlike }}(z)$ as obtained for $m=1, \bar{g}=1, \bar{\Phi}=1, L=3, \epsilon=10^{-10}$, and four choices of increasing $N$, in the affine scenario, for the fields $\bar{\phi}_{1}=$ $\rho \cos \theta$ and $\bar{\phi}_{2}=\rho \sin \theta$. The simulations, an order of magnitude shorter than the one of Fig. 2, rapidly converged and we had vanishing $\left\langle\overline{\phi_{i}}\right\rangle$ as required. From the figure we can see how the symmetry $z \rightarrow L-z$ appears to be broken in both two-point functions. In particular the unlike one appears to be oscillating close to the value of zero. This can be seen as an artifact due to the chosen asymmetric expression for the kinetic part of the primitive approximation. The two-point functions, that are now well converged, seem to have a well defined continuum limit $N \rightarrow \infty$. In fact the difference between $D_{\text {like }}(|z|=L / 2)$ from $N=10$ and $N=8$ is 0.043 but the one from $N=15$ and $N=13$ is 0.036 . This supports the conclusion that affine quantization leads to a well-defined field theory. This is also supported by looking at the renormalized mass and coupling constant [5]: $\quad m_{R}=0.101748(8), \bar{g}_{R}=1.50000(1)$ for $N=8$, $m_{R}=0.097307(8), \bar{g}_{R}=1.50000(2)$ for $N=10, m_{R}=$ $0.08949(4), \bar{g}_{R}=1.50000(3) \quad$ for $\quad N=13, \quad m_{R}=$ $0.08398(6), \bar{g}_{R}=1.49997(4)$ for $N=15$. We can see how the renormalized coupling constant remains constant upon the increase of $N$.

\section{CONCLUSIONS}

Summarizing, in this work we studied, through Monte Carlo simulations, the two-point function of a classical Euclidean covariant complex scalar field of mass $m$ subject to the Higgs Mexican-hat potential in four spacetime dimensions, treated either with canonical quantization and with affine quantization. And we analyzed the continuum limit at finite fixed volume. The finite volume constraint rules out the formation of the massless Goldstone boson due to the spontaneous symmetry breaking of the continuous phase symmetry $\varphi(x) \rightarrow e^{i \theta(x)} \varphi(x)$ that we continue to observe in the simulations even if only as a smooth transition (free energies in finite volume systems are always analytic).

We first studied the path integral in the two real fields $\phi_{1}$ and $\phi_{2}$ with $\varphi=\phi_{1}+i \phi_{2}$ through standard Metropolis [13] simulations. In the canonical case we found rapidly converging simulations: the unlike two-point function is zero everywhere and the like one shows the approach to the continuum through a diverging value at the origin. It is periodic of periodicity $L$ and satisfies the symmetry $z \rightarrow$ $L-z$ as it should. It has a minimum at half simulation box $|z|=L / 2$ close to zero, indicating that the scalar field 
theory is in the unbroken phase under canonical quantization, at the chosen couplings and dimension.

In the affine case we found that due to the appearance of the forbidden region around the origin $\varphi \approx 0$, the ergodicity of the random walk is broken. Once the field spontaneously breaks the symmetry falling in the circle of vacua, it can only rotate around the peak in the potential at the origin. Therefore very long simulations are necessary in order to find reliable results for the expectation values-more so approaching the continuum. This suggested to change variables from $\phi_{1}$ and $\phi_{2}$ to the modulus $\rho$ and the argument $\theta$ of the complex field, with $\varphi=\rho \exp (i \theta)$ and choose an asymmetric transition rule for the argument move in the Metropolis algorithm in order to allow only for counterclockwise rotations around the origin. This proved an effective way to overcome the ergodicity problem encountered previously, and the simulations converged quickly.
The approach to the continuum appears to be well behaved also for the affine case where the unlike twopoint function continues to be everywhere close to zero and the like one develops a minimum at half simulation box higher than the one observed in the canonical case indicating that the system under affine quantization is in the broken phase. Therefore we can say that affine quantization produces a meaningful quantum field theory. It would be interesting to carry on a detailed and systematic study of the approach to the continuum of the renormalized coupling constant in order to understand whether the affine approach is able to produce a nontrivial $[6,8-10,16]$ interacting field theory in the continuum limit also for the present case of a scalar complex field subject to the Higgs potential, as was done in our previous works for scalar real fields [4,5]. This would solve the problem of the believed triviality of the canonical Higgs particle in four space-time dimensions.
[1] J. R. Klauder, The benefits of affine quantization, J. High Energy Phys. Gravit. Cosmol. 6, 175 (2020).

[2] J. R. Klauder, Beyond Conventional Quantization (Cambridge University Press, Cambridge, England, 2000), Chap. 5.

[3] P. A. M. Dirac, The Principles of Quantum Mechanics (Clarendon Press, Oxford, 1958), in a footnote on page 114.

[4] R. Fantoni, Monte Carlo evaluation of the continuum limit of $\left(\phi^{12}\right)_{3}$, J. Stat. Mech. (2021) 083102.

[5] R. Fantoni and J. R. Klauder, Affine quantization of $\left(\varphi^{4}\right)_{4}$ succeeds while canonical quantization fails, Phys. Rev. D 103, 076013 (2021).

[6] J. Fröhlich, On the triviality of $\lambda \phi_{d}^{4}$ theories and the approach to the critical point in $d \geq 4$ dimensions, Nucl. Phys. B200, 281 (1982).

[7] M. Aizenman, Proof of the Triviality of $\phi_{d}^{4}$ Field Theory and Some Mean-Field Features of Ising Models for $d>4$, Phys. Rev. Lett. 47, 886(E) (1981).

[8] M. Lüscher and P. Weisz, Scaling laws and triviality bounds in the lattice $\phi^{4}$ theory: (I). One-component model in the symmetric phase, Nucl. Phys. B290, 25 (1987).

[9] M. Lüscher and P. Weisz, Scaling laws and triviality bounds in the lattice $\phi^{4}$ theory: (II). One-component model in the phase with spontaneous symmetry breaking, Nucl. Phys. B295, 65 (1988).

[10] M. Lüscher and P. Weisz, Scaling laws and triviality bounds in the lattice $\phi^{4}$ theory: (III). $n$-component model, Nucl. Phys. B318, 705 (1989).

[11] R. Fantoni and J. R. Klauder, Monte Carlo evaluation of the continuum limit of the two-point function of the Euclidean free real scalar field subject to affine quantization, J. Stat. Phys. 184, 28 (2021).

[12] M. H. Kalos and P. A. Whitlock, Monte Carlo Methods (Wiley-Vch Verlag GmbH \& Co., Germany, 2008).

[13] N. Metropolis, A. W. Rosenbluth, M. N. Rosenbluth, A. M. Teller, and E. Teller, Equation of state calculations by fast computing machines, J. Chem. Phys. 21, 1087 (1953).

[14] U. Wolff, Triviality of four dimensional $\phi^{4}$ theory on the lattice, Scholarpedia 9, 7367 (2014).

[15] D. M. Ceperley, Path integrals in the theory of condensed helium, Rev. Mod. Phys. 67, 279 (1995).

[16] K. G. Wilson and J. Kogut, The renormalization group and the $\epsilon$ expansion, Phys. Rep. 12, 75 (1974). 